\title{
Solving Optimization Problems on Hermitian Matrix Functions with Applications
}

\author{
Xiang Zhang and Shu-Wen Xiang \\ Department of Computer Science and Information, Guizhou University, Guiyang 550025, China \\ Correspondence should be addressed to Xiang Zhang; zxjnsc@163.com
}

Received 17 October 2012; Accepted 20 March 2013

Academic Editor: K. Sivakumar

Copyright (C) 2013 X. Zhang and S.-W. Xiang. This is an open access article distributed under the Creative Commons Attribution License, which permits unrestricted use, distribution, and reproduction in any medium, provided the original work is properly cited.

We consider the extremal inertias and ranks of the matrix expressions $f(X, Y)=A_{3}-B_{3} X-\left(B_{3} X\right)^{*}-C_{3} Y D_{3}-\left(C_{3} Y D_{3}\right)^{*}$, where $A_{3}=A_{3}^{*}, B_{3}, C_{3}$, and $D_{3}$ are known matrices and $Y$ and $X$ are the solutions to the matrix equations $A_{1} Y=C_{1}, Y B_{1}=D_{1}$, and $A_{2} X=C_{2}$, respectively. As applications, we present necessary and sufficient condition for the previous matrix function $f(X, Y)$ to be positive (negative), non-negative (positive) definite or nonsingular. We also characterize the relations between the Hermitian part of the solutions of the above-mentioned matrix equations. Furthermore, we establish necessary and sufficient conditions for the solvability of the system of matrix equations $A_{1} Y=C_{1}, Y B_{1}=D_{1}, A_{2} X=C_{2}$, and $B_{3} X+\left(B_{3} X\right)^{*}+C_{3} Y D_{3}+\left(C_{3} Y D_{3}\right)^{*}=A_{3}$, and give an expression of the general solution to the above-mentioned system when it is solvable.

\section{Introduction}

Throughout, we denote the field of complex numbers by $\mathbb{C}$, the set of all $m \times n$ matrices over $\mathbb{C}$ by $\mathbb{C}^{m \times n}$, and the set of all $m \times m$ Hermitian matrices by $\mathbb{C}_{h}^{m \times m}$. The symbols $A^{*}$ and $\mathscr{R}(A)$ stand for the conjugate transpose, the column space of a complex matrix $A$ respectively. $I_{n}$ denotes the $n \times n$ identity matrix. The Moore-Penrose inverse [1] $A^{\dagger}$ of $A$, is the unique solution $X$ to the four matrix equations:

$$
\begin{aligned}
& \text { (i) } A X A=A, \\
& \text { (ii) } X A X=X, \\
& \text { (iii) }(A X)^{*}=A X, \\
& \text { (iv) }(X A)^{*}=X A .
\end{aligned}
$$

Moreover, $L_{A}$ and $R_{A}$ stand for the projectors $L_{A}=I-$ $A^{\dagger} A, R_{A}=I-A A^{\dagger}$ induced by $A$. It is well known that the eigenvalues of a Hermitian matrix $A \in \mathbb{C}^{n \times n}$ are real, and the inertia of $A$ is defined to be the triplet

$$
\mathbb{\square}_{n}(A)=\left\{i_{+}(A), i_{-}(A), i_{0}(A)\right\},
$$

where $i_{+}(A), i_{-}(A)$, and $i_{0}(A)$ stand for the numbers of positive, negative, and zero eigenvalues of $A$, respectively. The symbols $i_{+}(A)$ and $i_{-}(A)$ are called the positive index and the negative index of inertia, respectively. For two Hermitian matrices $A$ and $B$ of the same sizes, we say $A \geq B(A \leq B)$ in the Löwner partial ordering if $A-B$ is positive (negative) semidefinite. The Hermitian part of $X$ is defined as $H(X)=$ $X+X^{*}$. We will say that $X$ is Re-nnd (Re-nonnegative semidefinite) if $H(X) \geq 0, X$ is Re-pd (Re-positive definite) if $H(X)>0$, and $X$ is Re-ns if $H(X)$ is nonsingular.

It is well known that investigation on the solvability conditions and the general solution to linear matrix equations is very active (e.g., [2-9]). In 1999, Braden [10] gave the general solution to

$$
B X+(B X)^{*}=A .
$$

In 2007, Djordjević [11] considered the explicit solution to (3) for linear bounded operators on Hilbert spaces. Moreover, Cao [12] investigated the general explicit solution to

$$
B X C+(B X C)^{*}=A .
$$

Xu et al. [13] obtained the general expression of the solution of operator equation (4). In 2012, Wang and He [14] studied 
some necessary and sufficient conditions for the consistence of the matrix equation

$$
A_{1} X+\left(A_{1} X\right)^{*}+B_{1} Y C_{1}+\left(B_{1} Y C_{1}\right)^{*}=E_{1}
$$

and presented an expression of the general solution to (5).

Note that (5) is a special case of the following system:

$$
\begin{aligned}
& A_{1} Y=C_{1}, \quad Y B_{1}=D_{1}, \quad A_{2} X=C_{2}, \\
& B_{3} X+\left(B_{3} X\right)^{*}+C_{3} Y D_{3}+\left(C_{3} Y D_{3}\right)^{*}=A_{3} .
\end{aligned}
$$

To our knowledge, there has been little information about (6). One goal of this paper is to give some necessary and sufficient conditions for the solvability of the system of matrix (6) and present an expression of the general solution to system (6) when it is solvable.

In order to find necessary and sufficient conditions for the solvability of the system of matrix equations (6), we need to consider the extremal ranks and inertias of (10) subject to (13) and (11).

There have been many papers to discuss the extremal ranks and inertias of the following Hermitian expressions:

$$
\begin{gathered}
p(X)=A_{3}-B_{3} X-\left(B_{3} X\right)^{*} \\
g(Y)=A-B Y C-(B Y C)^{*} \\
h(X, Y)=A_{1}-B_{1} X B_{1}^{*}-C_{1} Y C_{1}^{*} \\
f(X, Y)=A_{3}-B_{3} X-\left(B_{3} X\right)^{*}-C_{3} Y D_{3}-\left(C_{3} Y D_{3}\right)^{*} .
\end{gathered}
$$

Tian has contributed much in this field. One of his works [15] considered the extremal ranks and inertias of (7). $\mathrm{He}$ and Wang [16] derived the extremal ranks and inertias of (7) subject to $A_{1} X=C_{1}, A_{2} X B_{2}=C_{2}$. Liu and Tian [17] studied the extremal ranks and inertias of (8). Chu et al. [18] and Liu and Tian [19] derived the extremal ranks and inertias of (9). Zhang et al. [20] presented the extremal ranks and inertias of (9), where $X$ and $Y$ are Hermitian solutions of

$$
\begin{aligned}
& A_{2} X=C_{2}, \\
& Y B_{2}=D_{2},
\end{aligned}
$$

respectively. He and Wang [16] derived the extremal ranks and inertias of (10). We consider the extremal ranks and inertias of (10) subject to (11) and

$$
A_{1} Y=C_{1}, \quad Y B_{1}=D_{1},
$$

which is not only the generalization of the above matrix functions, but also can be used to investigate the solvability conditions for the existence of the general solution to the system (6). Moreover, it can be applied to characterize the relations between Hermitian part of the solutions of (11) and (13).

The remainder of this paper is organized as follows. In Section 2, we consider the extremal ranks and inertias of (10) subject to (11) and (13). In Section 3, we characterize the relations between the Hermitian part of the solution to (11) and (13). In Section 4, we establish the solvability conditions for the existence of a solution to (6) and obtain an expression of the general solution to (6).

\section{Extremal Ranks and Inertias of Hermitian Matrix Function (10) with Some Restrictions}

In this section, we consider formulas for the extremal ranks and inertias of (10) subject to (11) and (13). We begin with the following Lemmas.

Lemma 1 (see [21]). (a) Let $A_{1}, C_{1}, B_{1}$, and $D_{1}$ be given. Then the following statements are equivalent:

(1) system (13) is consistent,

(2)

$$
R_{A_{1}} C_{1}=0, \quad D_{1} L_{B_{1}}=0, \quad A_{1} D_{1}=C_{1} B_{1} .
$$

(3)

$$
\begin{gathered}
r\left[\begin{array}{ll}
A_{1} & C_{1}
\end{array}\right]=r\left(A_{1}\right), \\
{\left[\begin{array}{l}
D_{1} \\
B_{1}
\end{array}\right]=r\left(B_{1}\right),} \\
A_{1} D_{1}=C_{1} B_{1} .
\end{gathered}
$$

In this case, the general solution can be written as

$$
Y=A_{1}^{\dagger} C_{1}+L_{A_{1}} D_{1} B_{1}^{\dagger}+L_{A_{1}} V R_{B_{1}}
$$

where $V$ is arbitrary.

(b) Let $A_{2}$ and $C_{2}$ be given. Then the following statements are equivalent:

(1) equation (11) is consistent,

(2)

$$
R_{A_{2}} C_{2}=0
$$

(3)

$$
r\left[\begin{array}{ll}
A_{2} & C_{2}
\end{array}\right]=r\left(A_{2}\right) .
$$

In this case, the general solution can be written as

$$
X=A^{\dagger} C+L_{A} W
$$

where $W$ is arbitrary.

Lemma 2 ([22, Lemma 1.5, Theorem 2.3]). Let $A \in \mathbb{C}_{h}^{m \times m}$, $B \in \mathbb{C}^{m \times n}$, and $D \in \mathbb{C}_{h}^{n \times n}$, and denote that

$$
\begin{gathered}
M=\left[\begin{array}{cc}
A & B \\
B^{*} & 0
\end{array}\right], \\
N=\left[\begin{array}{cc}
0 & Q \\
Q^{*} & 0
\end{array}\right], \\
L=\left[\begin{array}{cc}
A & B \\
B^{*} & D
\end{array}\right], \\
G=\left[\begin{array}{cc}
P & M L_{N} \\
L_{N} M^{*} & 0
\end{array}\right] .
\end{gathered}
$$


Then one has the following

(a) the following equalities hold

$$
\begin{gathered}
i_{ \pm}(M)=r(B)+i_{ \pm}\left(R_{B} A R_{B}\right), \\
i_{ \pm}(N)=r(Q),
\end{gathered}
$$

(b) if $\mathscr{R}(B) \subseteq \mathscr{R}(A)$, then $i_{ \pm}(L)=i_{ \pm}(A)+i_{ \pm}(D-$ $\left.B^{*} A^{\dagger} B\right)$ ). Thus $i_{ \pm}(L)=i_{ \pm}(A)$ if and only if $\mathscr{R}(B) \subseteq$ $\mathscr{R}(A)$ and $i_{ \pm}\left(D-B^{*} A^{\dagger} B\right)=0$,

(c)

$$
i_{ \pm}(G)=\left[\begin{array}{ccc}
P & M & 0 \\
M^{*} & 0 & N^{*} \\
0 & N & 0
\end{array}\right]-r(N)
$$

Lemma 3 (see [23]). Let $A \in \mathbb{C}^{m \times n}, B \in \mathbb{C}^{m \times k}$, and $C \in \mathbb{C}^{l \times n}$. Then they satisfy the following rank equalities:
(a) $r\left[\begin{array}{ll}A & B\end{array}\right]=r(A)+r\left(E_{A} B\right)=r(B)+r\left(E_{B} A\right)$,
(b) $r\left[\begin{array}{l}A \\ C\end{array}\right]=r(A)+r\left(C F_{A}\right)=r(C)+r\left(A F_{C}\right)$,
(c) $r\left[\begin{array}{ll}A & B \\ C & 0\end{array}\right]=r(B)+r(C)+r\left(E_{B} A F_{C}\right)$,
(d) $r\left[\begin{array}{ll}B & A F_{C}\end{array}\right]=r\left[\begin{array}{ll}B & A \\ 0 & C\end{array}\right]-r(C)$,
(e) $r\left[\begin{array}{c}C \\ E_{B} A\end{array}\right]=r\left[\begin{array}{ll}C & 0 \\ A & B\end{array}\right]-r(B)$,
(f) $r\left[\begin{array}{cc}A & B F_{D} \\ E_{E} C & 0\end{array}\right]=r\left[\begin{array}{ccc}A & B & 0 \\ C & 0 & E \\ 0 & D & 0\end{array}\right]-r(D)-r(E)$,

Lemma 4 (see [15]). Let $A \in \mathbb{C}_{h}^{m \times m}, B \in \mathbb{C}^{m \times n}, C \in \mathbb{C}_{h}^{n \times n}, Q \in$ $\mathbb{C}^{m \times n}$, and $P \in \mathbb{C}^{p \times n}$ be given, and $T \in \mathbb{C}^{m \times m}$ be nonsingular. Then one has the following

(1) $i_{ \pm}\left(T A T^{*}\right)=i_{ \pm}(A)$,

(2) $i_{ \pm}\left[\begin{array}{ll}A & 0 \\ 0 & C\end{array}\right]=i_{ \pm}(A)+i_{ \pm}(C)$,

(3) $i_{ \pm}\left[\begin{array}{cc}0 & Q \\ Q^{*} & 0\end{array}\right]=r(Q)$,

(4) $i_{ \pm}\left[\begin{array}{cc}A & B L_{P} \\ L_{P} B^{*} & 0\end{array}\right]+r(P)=i_{ \pm}\left[\begin{array}{ccc}A & B & 0 \\ B^{*} & 0 & P^{*} \\ 0 & P & 0\end{array}\right]$

Lemma 5 (see [22, Lemma 1.4]). Let $S$ be a set consisting of (square) matrices over $\mathbb{C}^{m \times m}$, and let $H$ be a set consisting of (square) matrices over $\mathbb{C}_{h}^{m \times m}$. Then Then one has the following

(a) Shas a nonsingular matrix if and only if $\max _{X \in S} r(X)=$ $m$;

(b) any $X \in S$ is nonsingular if and only if $\min _{X \in S} r(X)=$ $m$;

(c) $\{0\} \in S$ if and only if $\min _{X \in S} r(X)=0$;

(d) $S=\{0\}$ if and only if $\max _{X \in S} r(X)=0$;

(e) $H$ has a matrix $X>0(X<0)$ if and only if $\max _{X \in H^{+}} i_{+}(X)=m\left(\max _{X \in H^{-}} i_{-}(X)=m\right)$;

(f) any $X \in H$ satisfies $X>0(X<0)$ if and only if $\min _{X \in H^{+}} i_{+}(X)=m\left(\min _{X \in H^{-}} i_{-}(X)=m\right)$;

(g) $H$ has a matrix $X \geq 0(X \leq 0)$ if and only if $\min _{X \in H^{i}} i_{-}(X)=0\left(\min _{X \in H^{+}} i_{+}(X)=0\right)$; (h) any $X \in H$ satisfies $X \geq 0(X \leq 0)$ if and only if $\max _{X \in H^{-}} i_{-}(X)=0\left(\max _{X \in H^{+}} i_{+}(X)=0\right)$.

Lemma 6 (see $[16])$. Let $p(X, Y)=A-B X-(B X)^{*}-C Y D-$ $(C Y D)^{*}$, where $A, B, C$, and $D$ are given with appropriate sizes, and denote that

$$
\begin{gathered}
M_{1}=\left[\begin{array}{ccc}
A & B & C \\
B^{*} & 0 & 0 \\
C^{*} & 0 & 0
\end{array}\right], \\
M_{2}=\left[\begin{array}{ccc}
A & B & D^{*} \\
B^{*} & 0 & 0 \\
D & 0 & 0
\end{array}\right], \\
M_{3}=\left[\begin{array}{cccc}
A & B & C & D^{*} \\
B^{*} & 0 & 0 & 0
\end{array}\right], \\
M_{4}=\left[\begin{array}{cccc}
A & B & C & D^{*} \\
B^{*} & 0 & 0 & 0 \\
C^{*} & 0 & 0 & 0
\end{array}\right], \\
M_{5}=\left[\begin{array}{cccc}
A & B & C & D^{*} \\
B^{*} & 0 & 0 & 0 \\
D & 0 & 0 & 0
\end{array}\right] .
\end{gathered}
$$

Then one has the following:

(1) the maximal rank of $p(X, Y)$ is

$$
\max _{X \in \mathbb{C}^{n \times m}, Y} r[p(X, Y)]=\min \left\{m, r\left(M_{1}\right), r\left(M_{2}\right), r\left(M_{3}\right)\right\},
$$

(2) the minimal rank of $p(X, Y)$ is

$$
\begin{aligned}
\min _{X \in \mathbb{C}^{n \times m}, Y} r[p(X, Y)] \\
=2 r\left(M_{3}\right)-2 r(B) \\
\quad+\max \left\{u_{+}+u_{-}, v_{+}+v_{-}, u_{+}+v_{-}, u_{-}+v_{+}\right\},
\end{aligned}
$$

(3) the maximal inertia of $p(X, Y)$ is

$$
\max _{X \in \mathbb{C}^{n \times m}, Y} i_{ \pm}[p(X, Y)]=\min \left\{i_{ \pm}\left(M_{1}\right), i_{ \pm}\left(M_{2}\right)\right\},
$$

(4) the minimal inertias of $p(X, Y)$ is

$$
\begin{aligned}
\min _{X \in \mathbb{C}^{n \times m}, Y} i_{ \pm}[p(X, Y)]= & r\left(M_{3}\right)-r(B) \\
& +\max \left\{i_{ \pm}\left(M_{1}\right)-r\left(M_{4}\right),\right. \\
& \left.i_{ \pm}\left(M_{2}\right)-r\left(M_{5}\right)\right\},
\end{aligned}
$$

where

$$
u_{ \pm}=i_{ \pm}\left(M_{1}\right)-r\left(M_{4}\right), \quad v_{ \pm}=i_{ \pm}\left(M_{2}\right)-r\left(M_{5}\right) .
$$

Now we present the main theorem of this section. 
Theorem 7. Let $A_{1} \in \mathbb{C}^{m \times n}, C_{1} \in \mathbb{C}^{m \times k}, B_{1} \in \mathbb{C}^{k \times l}, D_{1} \in \mathbb{C}^{n \times l}$, $A_{2} \in \mathbb{C}^{t \times q}, C_{2} \in \mathbb{C}^{t \times p}, A_{3} \in \mathbb{C}_{h}^{p \times p}, B_{3} \in \mathbb{C}^{p \times q}, C_{3} \in \mathbb{C}^{p \times n}$, and $D_{3} \in \mathbb{C}^{p \times n}$ be given, and suppose that the system of matrix equations (13) and (11) is consistent, respectively. Denote the set of all solutions to (13) by S and (11) by G. Put

$$
\begin{aligned}
& E_{1}=\left[\begin{array}{ccccc}
A_{3} & C_{3} & D_{3}^{*} C_{1}^{*} & B_{3} & C_{2}^{*} \\
C_{3}^{*} & 0 & A_{1}^{*} & 0 & 0 \\
C_{1} D_{3} & A_{1} & 0 & 0 & 0 \\
B_{3}^{*} & 0 & 0 & 0 & A_{2}^{*} \\
C_{2} & 0 & 0 & A_{2} & 0
\end{array}\right], \\
& E_{2}=r\left[\begin{array}{ccccc}
A_{3} & D_{3}^{*} & C_{3} D_{1} & B_{3} & C_{2}^{*} \\
D_{3} & 0 & B_{1} & 0 & 0 \\
D_{1}^{*} C_{3}^{*} & B_{1}^{*} & 0 & 0 & 0 \\
B_{3}^{*} & 0 & 0 & 0 & A_{2}^{*} \\
C_{2} & 0 & 0 & A_{2} & 0
\end{array}\right], \\
& E_{3}=\left[\begin{array}{ccccc}
A_{3} & B_{3} & C_{3}^{*} & D_{3}^{*} & C_{2}^{*} \\
B_{3}^{*} & 0 & 0 & 0 & A_{2}^{*} \\
D_{1}^{*} C_{3}^{*} & 0 & 0 & B_{1}^{*} & 0 \\
C_{1} D_{3} & 0 & A_{1} & 0 & 0 \\
C_{2} & A_{2} & 0 & 0 & 0
\end{array}\right], \\
& E_{4}=\left[\begin{array}{cccccc}
A_{3} & B_{3} & C_{3} & D_{3}^{*} & C_{2}^{*} & D_{3}^{*} C_{1}^{*} \\
B_{3}^{*} & 0 & 0 & 0 & A_{2}^{*} & 0 \\
C_{3}^{*} & 0 & 0 & 0 & 0 & A_{1}^{*} \\
0 & 0 & 0 & B_{1}^{*} & 0 & 0 \\
C_{1} D_{3} & 0 & A_{1} & 0 & 0 & 0 \\
C_{2} & A_{2} & 0 & 0 & 0 & 0
\end{array}\right] \\
& E_{5}=\left[\begin{array}{cccccc}
A_{3} & B_{3} & C_{3} & D_{3}^{*} & C_{2}^{*} & C_{3} D_{1} \\
B_{3}^{*} & 0 & 0 & 0 & A_{2}^{*} & 0 \\
D_{3} & 0 & 0 & 0 & 0 & B_{1} \\
D_{1}^{*} C_{3}^{*} & 0 & 0 & A_{1}^{*} & 0 & 0 \\
0 & 0 & A_{1} & 0 & 0 & 0 \\
C_{2} & A_{2} & 0 & 0 & 0 & 0
\end{array}\right]
\end{aligned}
$$

Then one has the following:

(a) the maximal rank of (10) subject to (13) and (11) is

$$
\begin{aligned}
& \max _{X \in G, Y \in S} r[f(X, Y)] \\
&=\min \left\{p, r\left(E_{1}\right)-2 r\left(A_{1}\right)-2 r\left(A_{2}\right),\right. \\
& r\left(E_{2}\right)-2 r\left(B_{1}\right)-2 r\left(A_{2}\right), \\
&\left.r\left(E_{3}\right)-2 r\left(A_{2}\right)-r\left(A_{1}\right)-r\left(B_{1}\right)\right\},
\end{aligned}
$$

(b) the minimal rank of (10) subject to (13) and (11) is

$$
\begin{aligned}
\min _{X \in G, Y \in S} r[f(X, Y)] & \\
= & 2 r\left(E_{3}\right)-2 r\left[\begin{array}{l}
B_{3} \\
A_{2}
\end{array}\right] \\
+ & \max \left\{r\left(E_{1}\right)-2 r\left(E_{4}\right), r\left(E_{2}\right)-2 r\left(E_{5}\right),\right. \\
& i_{+}\left(E_{1}\right)+i_{-}\left(E_{2}\right)-r\left(E_{4}\right)-r\left(E_{5}\right), \\
& \left.i_{-}\left(E_{1}\right)+i_{+}\left(E_{2}\right)-r\left(E_{4}\right)-r\left(E_{5}\right)\right\},
\end{aligned}
$$

(c) the maximal inertia of (10) subject to (13) and (11) is

$$
\begin{array}{r}
\max _{X \in G, Y \in S x} i_{ \pm}[f(X, Y)]=\min \left\{i_{ \pm}\left(E_{1}\right)-r\left(A_{1}\right)-r\left(A_{2}\right),\right. \\
\left.i_{ \pm}\left(E_{2}\right)-r\left(B_{1}\right)-r\left(A_{2}\right)\right\},
\end{array}
$$

(d) the minimal inertia of (10) subject to (13) and (11) is

$$
\begin{aligned}
\min _{X \in G, Y \in S_{ \pm}} i_{ \pm}[f(X, Y)]= & r\left(E_{3}\right)-r\left[\begin{array}{c}
B_{3} \\
A_{2}
\end{array}\right] \\
& +\max \left\{i_{ \pm}\left(E_{1}\right)-r\left(E_{4}\right),\right. \\
& \left.i_{ \pm}\left(E_{2}\right)-r\left(E_{5}\right)\right\} .
\end{aligned}
$$

Proof. By Lemma 1, the general solutions to (13) and (11) can be written as

$$
\begin{gathered}
X=A_{2}^{\dagger} C_{2}+L_{A_{2}} W \\
Y=A_{1}^{\dagger} C_{1}+L_{A_{1}} D_{1} B_{1}^{\dagger}+L_{A_{1}} Z R_{B_{1}},
\end{gathered}
$$

where $W$ and $Z$ are arbitrary matrices with appropriate sizes. Put

$$
\begin{gathered}
Q=B_{3} L_{A_{2}}, \quad T=C_{3} L_{A_{1}}, \quad J=R_{B_{1}} D_{3}, \\
P=A_{3}-B_{3} A_{2}^{\dagger} C_{2}-\left(B_{3} A_{2}^{\dagger} C_{2}\right)^{*} \\
-C_{3}\left(A_{1}^{\dagger} C_{1}+L_{A_{1}} D_{1} B_{1}^{\dagger}\right) D_{3} \\
-\left(C_{3}\left(A_{1}^{\dagger} C_{1}+L_{A_{1}} D_{1} B_{1}^{\dagger}\right) D_{3}\right)^{*} .
\end{gathered}
$$

Substituting (36) into (10) yields

$$
f(X, Y)=P-Q W-(Q W)^{*}-T Z J-(T Z J)^{*}
$$

Clearly $P$ is Hermitian. It follows from Lemma 6 that

$$
\begin{aligned}
\max _{X \in G, Y \in S} r[f(X, Y)] \\
=\max _{W, Z} r\left(P-Q W-(Q W)^{*}-T Z J-(T Z J)^{*}\right) \\
=\min \left\{m, r\left(N_{1}\right), r\left(N_{2}\right), r\left(N_{3}\right)\right\}, \\
\min _{X \in G, Y \in S} r[f(X, Y)] \\
=\max _{W, Z} r\left(P-Q W-(Q W)^{*}-T Z J-(T Z J)^{*}\right) \\
=2 r\left(N_{3}\right)-2 r(Q) \\
\quad+\max \left\{s_{+}+s_{-}, t_{+}+t_{-}, s_{+}+t_{-}, s_{-}+t_{+}\right\},
\end{aligned}
$$




$$
\begin{aligned}
& \max _{X \in G, Y \in S} i_{ \pm}[f(X, Y)] \\
& =\max _{W, Z} r\left(P-Q W-(Q W)^{*}-T Z J-(T Z J)^{*}\right) \\
& =\min \left\{i_{ \pm}\left(N_{1}\right), i_{ \pm}\left(N_{2}\right)\right\}, \\
& \min _{X \in G, Y \in S^{+}} i_{ \pm}[f(X, Y)] \\
& =\max _{W, Z} r\left(P-Q W-(Q W)^{*}-T Z J-(T Z J)^{*}\right) \\
& =r\left(N_{3}\right)-r(Q)+\max \left\{s_{ \pm}, t_{ \pm}\right\},
\end{aligned}
$$

where

$$
\begin{aligned}
N_{1} & =\left[\begin{array}{ccc}
P & Q & T \\
Q^{*} & 0 & 0 \\
T^{*} & 0 & 0
\end{array}\right], \\
N_{2} & =\left[\begin{array}{ccc}
P & Q & J^{*} \\
Q^{*} & 0 & 0 \\
J & 0 & 0
\end{array}\right], \\
N_{3} & =\left[\begin{array}{cccc}
P & Q & T & J^{*} \\
Q^{*} & 0 & 0 & 0
\end{array}\right], \\
N_{4} & =\left[\begin{array}{cccc}
P & Q & T & J^{*} \\
Q^{*} & 0 & 0 & 0 \\
T^{*} & 0 & 0 & 0
\end{array}\right], \\
N_{5} & =\left[\begin{array}{cccc}
P & Q & T & J^{*} \\
Q^{*} & 0 & 0 & 0 \\
J & 0 & 0 & 0
\end{array}\right], \\
s_{ \pm}=i_{ \pm}\left(N_{1}\right)-r & \left(N_{4}\right), \quad t_{ \pm}=i_{ \pm}\left(N_{2}\right)-r\left(N_{5}\right) .
\end{aligned}
$$

Now, we simplify the ranks and inertias of block matrices in (38)-(41).

By Lemma 4, block Gaussian elimination, and noting that

$$
L_{S}^{*}=\left(I-S^{\dagger} S\right)^{*}=I-S^{*}\left(S^{*}\right)^{\dagger}=R_{S^{*}},
$$

we have the following:

$$
\begin{aligned}
r\left(N_{1}\right)=r & {\left[\begin{array}{ccc}
P & Q & T \\
Q^{*} & 0 & 0 \\
T^{*} & 0 & 0
\end{array}\right] } \\
= & {\left[\begin{array}{ccccc}
A_{3} & C_{3} & D_{3}^{*} C_{1}^{*} & B_{3} & C_{2}^{*} \\
C_{3}^{*} & 0 & A_{1}^{*} & 0 & 0 \\
C_{1} D_{3} & A_{1} & 0 & 0 & 0 \\
B_{3}^{*} & 0 & 0 & 0 & A_{2}^{*} \\
C_{2} & 0 & 0 & A_{2} & 0
\end{array}\right] } \\
& -2 r\left(A_{1}\right)-2 r\left(A_{2}\right) .
\end{aligned}
$$

By $C_{1} B_{1}=A_{1} D_{1}$, we obtain

$$
r\left(N_{2}\right)=r\left[\begin{array}{ccc}
P & Q & J^{*} \\
Q^{*} & 0 & 0 \\
J & 0 & 0
\end{array}\right]
$$$$
=r\left[\begin{array}{ccccc}
A_{3} & D_{3}^{*} & C_{3} D_{1} & B_{3} & C_{2}^{*} \\
D_{3} & 0 & B_{1} & 0 & 0 \\
D_{1}^{*} C_{3}^{*} & B_{1}^{*} & 0 & 0 & 0 \\
B_{3}^{*} & 0 & 0 & 0 & A_{2}^{*} \\
C_{2} & 0 & 0 & A_{2} & 0
\end{array}\right]
$$$$
-2 r\left(B_{1}\right)-2 r\left(A_{2}\right) \text {, }
$$

$$
r\left(N_{3}\right)=r\left[\begin{array}{cccc}
P & Q & T & J^{*} \\
Q^{*} & 0 & 0 & 0
\end{array}\right]
$$$$
=r\left[\begin{array}{ccccc}
A_{3} & B_{3} & C_{3}^{*} & D_{3}^{*} & C_{2}^{*} \\
B_{3}^{*} & 0 & 0 & 0 & A_{2}^{*} \\
D_{1}^{*} C_{3}^{*} & 0 & 0 & B_{1}^{*} & 0 \\
C_{1} D_{3} & 0 & A_{1} & 0 & 0 \\
C_{2} & A_{2} & 0 & 0 & 0
\end{array}\right]
$$$$
-r\left(B_{1}\right)-2 r\left(A_{2}\right)-r\left(A_{1}\right) \text {, }
$$$$
r\left(N_{4}\right)=r\left[\begin{array}{cccc}
P & Q & T & J^{*} \\
Q^{*} & 0 & 0 & 0 \\
T^{*} & 0 & 0 & 0
\end{array}\right]
$$$$
=r\left[\begin{array}{cccccc}
A_{3} & B_{3} & C_{3} & D_{3}^{*} & C_{2}^{*} & D_{3}^{*} C_{1}^{*} \\
B_{3}^{*} & 0 & 0 & 0 & A_{2}^{*} & 0 \\
C_{3}^{*} & 0 & 0 & 0 & 0 & A_{1}^{*} \\
0 & 0 & 0 & B_{1}^{*} & 0 & 0 \\
C_{1} D_{3} & 0 & A_{1} & 0 & 0 & 0 \\
C_{2} & A_{2} & 0 & 0 & 0 & 0
\end{array}\right]
$$$$
-r\left(B_{1}\right)-2 r\left(A_{2}\right)-2 r\left(A_{1}\right),
$$

$$
r\left(N_{5}\right)=r\left[\begin{array}{cccc}
P & Q & T & J^{*} \\
Q^{*} & 0 & 0 & 0 \\
T^{*} & 0 & 0 & 0
\end{array}\right]
$$

$$
\begin{gathered}
=r\left[\begin{array}{cccccc}
A_{3} & B_{3} & C_{3} & D_{3}^{*} & C_{2}^{*} & C_{3} D_{1} \\
B_{3}^{*} & 0 & 0 & 0 & A_{2}^{*} & 0 \\
D_{3} & 0 & 0 & 0 & 0 & B_{1} \\
D_{1}^{*} C_{3}^{*} & 0 & 0 & A_{1}^{*} & 0 & 0 \\
0 & 0 & A_{1} & 0 & 0 & 0 \\
C_{2} & A_{2} & 0 & 0 & 0 & 0
\end{array}\right] \\
-2 r\left(B_{1}\right)-2 r\left(A_{2}\right)-r\left(A_{1}\right) .
\end{gathered}
$$

By Lemma 2, we can get the following:

$$
i_{ \pm}\left(N_{1}\right)=i_{ \pm}\left[\begin{array}{ccc}
P & Q & T \\
Q^{*} & 0 & 0 \\
T^{*} & 0 & 0
\end{array}\right]
$$




$$
\begin{aligned}
= & i_{ \pm}\left[\begin{array}{ccccc}
A_{3} & C_{3} & D_{3}^{*} C_{1}^{*} & B_{3} & C_{2}^{*} \\
C_{3}^{*} & 0 & A_{1}^{*} & 0 & 0 \\
C_{1} D_{3} & A_{1} & 0 & 0 & 0 \\
B_{3}^{*} & 0 & 0 & 0 & A_{2}^{*} \\
C_{2} & 0 & 0 & A_{2} & 0
\end{array}\right] \\
i_{ \pm}\left(N_{2}\right)= & -r\left(A_{1}\right)-r\left(A_{2}\right), \\
& {\left[\begin{array}{ccc}
P & Q & J^{*} \\
Q^{*} & 0 & 0 \\
J & 0 & 0
\end{array}\right] } \\
& -\left[\begin{array}{ccccc}
A_{3} & D_{3}^{*} & C_{3} D_{1} & B_{3} & C_{2}^{*} \\
D_{3} & 0 & B_{1} & 0 & 0 \\
D_{1}^{*} C_{3}^{*} & B_{1}^{*} & 0 & 0 & 0 \\
B_{3}^{*} & 0 & 0 & 0 & A_{2}^{*} \\
C_{2} & 0 & 0 & A_{2} & 0
\end{array}\right] \\
& -r\left(B_{1}\right)-r\left(A_{2}\right) .
\end{aligned}
$$

Substituting (44)-(47) into (38) and (41) yields (31)-(34), respectively.

Corollary 8. Let $A_{1}, C_{1}, B_{1}, D_{1}, A_{2}, C_{2}, A_{3}, B_{3}, C_{3}, D_{3}$, and $E_{i},(i=1,2, \ldots, 5)$ be as in Theorem 7 , and suppose that the system of matrix equations (13) and (11) is consistent, respectively. Denote the set of all solutions to (13) by $S$ and (11) by $G$. Then, one has the following:

(a) there exist $X \in G$ and $Y \in S$ such that $A_{3}-B_{3} X-$ $\left(B_{3} X\right)^{*}-C_{3} Y D_{3}-\left(C_{3} Y D_{3}\right)^{*}>0$ if and only if

$$
\begin{aligned}
& i_{+}\left(E_{1}\right)-r\left(A_{1}\right)-r\left(A_{2}\right) \geq p, \\
& i_{+}\left(E_{2}\right)-r\left(B_{1}\right)-r\left(A_{2}\right) \geq p .
\end{aligned}
$$

(b) there exist $X \in G$ and $Y \in S$ such that $A_{3}-B_{3} X-$ $\left(B_{3} X\right)^{*}-C_{3} Y D_{3}-\left(C_{3} Y D_{3}\right)^{*}<0$ if and only if

$$
\begin{aligned}
& i_{-}\left(E_{1}\right)-r\left(A_{1}\right)-r\left(A_{2}\right) \geq p, \\
& i_{-}\left(E_{2}\right)-r\left(B_{1}\right)-r\left(A_{2}\right) \geq p,
\end{aligned}
$$

(c) there exist $X \in G$ and $Y \in S$ such that $A_{3}-B_{3} X-$ $\left(B_{3} X\right)^{*}-C_{3} Y D_{3}-\left(C_{3} Y D_{3}\right)^{*} \geq 0$ if and only if

$$
\begin{aligned}
& r\left(E_{3}\right)-r\left[\begin{array}{l}
B_{3} \\
A_{2}
\end{array}\right]+i_{-}\left(E_{1}\right)-r\left(E_{4}\right) \leq 0, \\
& r\left(E_{3}\right)-r\left[\begin{array}{l}
B_{3} \\
A_{2}
\end{array}\right]+i_{-}\left(E_{2}\right)-r\left(E_{5}\right) \leq 0 .
\end{aligned}
$$

(d) there exist $X \in G$ and $Y \in S$ such that $A_{3}-B_{3} X-$ $\left(B_{3} X\right)^{*}-C_{3} Y D_{3}-\left(C_{3} Y D_{3}\right)^{*} \leq 0$ if and only if

$$
\begin{aligned}
& r\left(E_{3}\right)-r\left[\begin{array}{l}
B_{3} \\
A_{2}
\end{array}\right]+i_{+}\left(E_{1}\right)-r\left(E_{4}\right) \leq 0, \\
& r\left(E_{3}\right)-r\left[\begin{array}{l}
B_{3} \\
A_{2}
\end{array}\right]+i_{+}\left(E_{2}\right)-r\left(E_{5}\right) \leq 0,
\end{aligned}
$$

(e) $A_{3}-B_{3} X-\left(B_{3} X\right)^{*}-C_{3} Y D_{3}-\left(C_{3} Y D_{3}\right)^{*}>0$ for all $X \in G$ and $Y \in S$ if and only if

$$
\begin{aligned}
& r\left(E_{3}\right)-r\left[\begin{array}{l}
B_{3} \\
A_{2}
\end{array}\right]+i_{+}\left(E_{1}\right)-r\left(E_{4}\right)=p \\
& \quad \text { or } \quad r\left(E_{3}\right)-r\left[\begin{array}{l}
B_{3} \\
A_{2}
\end{array}\right]+i_{+}\left(E_{2}\right)-r\left(E_{5}\right)=p,
\end{aligned}
$$

(f) $A_{3}-B_{3} X-\left(B_{3} X\right)^{*}-C_{3} Y D_{3}-\left(C_{3} Y D_{3}\right)^{*}<0$ for all $X \in G$ and $Y \in S$ if and only if

$$
\begin{aligned}
& r\left(E_{3}\right)-r\left[\begin{array}{l}
B_{3} \\
A_{2}
\end{array}\right]+i_{-}\left(E_{1}\right)-r\left(E_{4}\right)=p \\
& \quad \text { or } \quad r\left(E_{3}\right)-r\left[\begin{array}{l}
B_{3} \\
A_{2}
\end{array}\right]+i_{-}\left(E_{2}\right)-r\left(E_{5}\right)=p,
\end{aligned}
$$

(g) $A_{3}-B_{3} X-\left(B_{3} X\right)^{*}-C_{3} Y D_{3}-\left(C_{3} Y D_{3}\right)^{*} \geq 0$ for all $X \in G$ and $Y \in S$ if and only if

$$
\begin{aligned}
& i_{-}\left(E_{1}\right)-r\left(A_{1}\right)-r\left(A_{2}\right) \leq 0 \\
& \quad \text { or } \quad i_{-}\left(E_{2}\right)-r\left(B_{1}\right)-r\left(A_{2}\right) \leq 0,
\end{aligned}
$$

(h) $A_{3}-B_{3} X-\left(B_{3} X\right)^{*}-C_{3} Y D_{3}-\left(C_{3} Y D_{3}\right)^{*} \leq 0$ for all $X \in G$ and $Y \in S$ if and only if

$$
\begin{aligned}
& i_{+}\left(E_{1}\right)-r\left(A_{1}\right)-r\left(A_{2}\right) \leq 0 \\
& \quad \text { or } \quad i_{+}\left(E_{2}\right)-r\left(B_{1}\right)-r\left(A_{2}\right) \leq 0,
\end{aligned}
$$

(i) there exist $X \in G$ and $Y \in S$ such that $A_{3}-B_{3} X-$ $\left(B_{3} X\right)^{*}-C_{3} Y D_{3}-\left(C_{3} Y D_{3}\right)^{*}$ is nonsingular if and only if

$$
\begin{gathered}
r\left(E_{1}\right)-2 r\left(A_{1}\right)-2 r\left(A_{2}\right) \geq p, \\
r\left(E_{2}\right)-2 r\left(B_{1}\right)-2 r\left(A_{2}\right) \geq p, \\
r\left(E_{3}\right)-2 r\left(A_{2}\right)-r\left(A_{1}\right)-r\left(B_{1}\right) \geq p .
\end{gathered}
$$

\section{Relations between the Hermitian Part of the Solutions to (13) and (11)}

Now we consider the extremal ranks and inertias of the difference between the Hermitian part of the solutions to (13) and (11).

Theorem 9. Let $A_{1} \in \mathbb{C}^{m \times p}, C_{1} \in \mathbb{C}^{m \times p}, B_{1} \in \mathbb{C}^{p \times l}, D_{1} \in$ $\mathbb{C}^{p \times l}, A_{2} \in \mathbb{C}^{t \times p}$, and $C_{2} \in \mathbb{C}^{t \times p}$, be given. Suppose that the system of matrix equations (13) and (11) is consistent, 
respectively. Denote the set of all solutions to (13) by $S$ and (11) by G. Put

$$
\begin{aligned}
& H_{1}=\left[\begin{array}{ccccc}
0 & I & C_{1}^{*} & -I & C_{2}^{*} \\
I & 0 & A_{1}^{*} & 0 & 0 \\
C_{1} & A_{1} & 0 & 0 & 0 \\
-I & 0 & 0 & 0 & A_{2}^{*} \\
C_{2} & 0 & 0 & A_{2} & 0
\end{array}\right], \\
& H_{2}=r\left[\begin{array}{ccccc}
0 & I & D_{1} & -I & C_{2}^{*} \\
I & 0 & B_{1} & 0 & 0 \\
D_{1}^{*} & B_{1}^{*} & 0 & 0 & 0 \\
-I & 0 & 0 & 0 & A_{2}^{*} \\
C_{2} & 0 & 0 & A_{2} & 0
\end{array}\right] \text {, } \\
& H_{3}=\left[\begin{array}{ccccc}
0 & -I & I & I & C_{2}^{*} \\
-I & 0 & 0 & 0 & A_{2}^{*} \\
D_{1}^{*} & 0 & 0 & B_{1}^{*} & 0 \\
C_{1} & 0 & A_{1} & 0 & 0 \\
C_{2} & A_{2} & 0 & 0 & 0
\end{array}\right] \\
& H_{4}=\left[\begin{array}{cccccc}
0 & -I & I & I & C_{2}^{*} & C_{1}^{*} \\
-I & 0 & 0 & 0 & A_{2}^{*} & 0 \\
I & 0 & 0 & 0 & 0 & A_{1}^{*} \\
0 & 0 & 0 & B_{1}^{*} & 0 & 0 \\
C_{1} & 0 & A_{1} & 0 & 0 & 0 \\
C_{2} & A_{2} & 0 & 0 & 0 & 0
\end{array}\right] \text {, } \\
& H_{5}=\left[\begin{array}{cccccc}
0 & -I & I & I & C_{2}^{*} & D_{1} \\
-I & 0 & 0 & 0 & A_{2}^{*} & 0 \\
I & 0 & 0 & 0 & 0 & B_{1} \\
D_{1}^{*} & 0 & 0 & A_{1}^{*} & 0 & 0 \\
0 & 0 & A_{1} & 0 & 0 & 0 \\
C_{2} & A_{2} & 0 & 0 & 0 & 0
\end{array}\right] \text {. }
\end{aligned}
$$

Then one has the following:

$$
\begin{aligned}
& \max _{X \in G, Y \in S} r {\left[\left(X+X^{*}\right)-\left(Y+Y^{*}\right)\right] } \\
&=\min \left\{p, r\left(H_{1}\right)-2 r\left(A_{1}\right)-2 r\left(A_{2}\right), r\left(H_{2}\right)-2 r\left(B_{1}\right)\right.\left.-2 r\left(A_{2}\right), r\left(H_{3}\right)-2 r\left(A_{2}\right)-r\left(A_{1}\right)-r\left(B_{1}\right)\right\}, \\
& \min _{X \in G, Y \in S} r\left[\left(X+X^{*}\right)-\left(Y+Y^{*}\right)\right] \\
&=2 r\left(H_{3}\right)-2 p \\
&+\max \left\{r\left(H_{1}\right)-2 r\left(H_{4}\right), r\left(H_{2}\right)-2 r\left(H_{5}\right),\right. \\
& \quad i_{+}\left(H_{1}\right)+i_{-}\left(H_{2}\right)-r\left(H_{4}\right)-r\left(H_{5}\right), \\
&\left.\quad i_{-}\left(H_{1}\right)+i_{+}\left(H_{2}\right)-r\left(H_{4}\right)-r\left(H_{5}\right)\right\}, \\
& \max _{X \in G, Y \in S} i_{ \pm}\left[\left(X+X^{*}\right)-\left(Y+Y^{*}\right)\right] \\
&=\min \left\{i_{ \pm}\left(H_{1}\right)-r\left(A_{1}\right)-r\left(A_{2}\right),\right. \\
&\left.\quad i_{ \pm}\left(H_{2}\right)-r\left(B_{1}\right)-r\left(A_{2}\right)\right\},
\end{aligned}
$$

$$
\begin{aligned}
& \min _{X \in G, Y \in S} i_{ \pm}\left[\left(X+X^{*}\right)-\left(Y+Y^{*}\right)\right] \\
& \quad=r\left(E_{3}\right)-p+\max \left\{i_{ \pm}\left(H_{1}\right)-r\left(H_{4}\right), i_{ \pm}\left(H_{2}\right)-r\left(H_{5}\right)\right\} .
\end{aligned}
$$

Proof. By letting $A_{3}=0, B_{3}=-I, C_{3}=I$, and $D_{3}=I$ in Theorem 7, we can get the results.

Corollary 10. Let $A_{1}, C_{1}, B_{1}, D_{1}, A_{2}, C_{2}$, and $H_{i}, \quad(i=$ $1,2, \ldots, 5)$ be as in Theorem 9, and suppose that the system of matrix equations (13) and (11) is consistent, respectively. Denote the set of all solutions to (13) by $S$ and (11) by G. Then, one has the following:

(a) there exist $X \in G$ and $Y \in S$ such that $\left(X+X^{*}\right)>$ $\left(Y+Y^{*}\right)$ if and only if

$$
\begin{aligned}
& i_{+}\left(H_{1}\right)-r\left(A_{1}\right)-r\left(A_{2}\right) \geq p, \\
& i_{+}\left(H_{2}\right)-r\left(B_{1}\right)-r\left(A_{2}\right) \geq p .
\end{aligned}
$$

(b) there exist $X \in G$ and $Y \in S$ such that $\left(X+X^{*}\right)<$ $\left(Y+Y^{*}\right)$ if and only if

$$
\begin{aligned}
& i_{-}\left(H_{1}\right)-r\left(A_{1}\right)-r\left(A_{2}\right) \geq p, \\
& i_{-}\left(H_{2}\right)-r\left(B_{1}\right)-r\left(A_{2}\right) \geq p,
\end{aligned}
$$

(c) there exist $X \in G$ and $Y \in S$ such that $\left(X+X^{*}\right) \geq$ $\left(Y+Y^{*}\right)$ if and only if

$$
\begin{aligned}
& r\left(H_{3}\right)-p+i_{-}\left(H_{1}\right)-r\left(H_{4}\right) \leq 0, \\
& r\left(H_{3}\right)-p+i_{-}\left(H_{1}\right)-r\left(H_{4}\right) \leq 0,
\end{aligned}
$$

(d) there exist $X \in G$ and $Y \in S$ such that $\left(X+X^{*}\right) \leq$ $\left(Y+Y^{*}\right)$ if and only if

$$
\begin{aligned}
& r\left(H_{3}\right)-p+i_{+}\left(H_{1}\right)-r\left(H_{4}\right) \leq 0, \\
& r\left(H_{3}\right)-p+i_{+}\left(H_{2}\right)-r\left(H_{5}\right) \leq 0,
\end{aligned}
$$

(e) $\left(X+X^{*}\right)>\left(Y+Y^{*}\right)$ for all $X \in G$ and $Y \in S$ if and only if

$$
\begin{aligned}
& r\left(H_{3}\right)-p+i_{+}\left(H_{1}\right)-r\left(H_{4}\right)=p \\
& \quad \text { or } \quad r\left(H_{3}\right)-p+i_{+}\left(H_{2}\right)-r\left(H_{5}\right)=p,
\end{aligned}
$$

(f) $\left(X+X^{*}\right)<\left(Y+Y^{*}\right)$ for all $X \in G$ and $Y \in S$ if and only if

$$
\begin{aligned}
& r\left(H_{3}\right)-p+i_{-}\left(H_{1}\right)-r\left(H_{4}\right)=p \\
& \quad \text { or } \quad r\left(H_{3}\right)-p+i_{-}\left(H_{2}\right)-r\left(H_{5}\right)=p,
\end{aligned}
$$

(g) $\left(X+X^{*}\right) \geq\left(Y+Y^{*}\right)$ for all $X \in G$ and $Y \in S$ if and only if

$$
\begin{aligned}
& i_{-}\left(H_{1}\right)-r\left(A_{1}\right)-r\left(A_{2}\right) \leq 0 \\
& \quad \text { or } \quad i_{-}\left(H_{2}\right)-r\left(B_{1}\right)-r\left(A_{2}\right) \leq 0,
\end{aligned}
$$


(h) $\left(X+X^{*}\right) \leq\left(Y+Y^{*}\right)$ for all $X \in G$ and $Y \in S$ if and only if

$$
\begin{aligned}
& i_{+}\left(H_{1}\right)-r\left(A_{1}\right)-r\left(A_{2}\right) \leq 0 \\
& \quad \text { or } \quad i_{+}\left(H_{2}\right)-r\left(B_{1}\right)-r\left(A_{2}\right) \leq 0,
\end{aligned}
$$

(i) there exist $X \in G$ and $Y \in S$ such that $\left(X+X^{*}\right)-(Y+$ $\left.Y^{*}\right)$ is nonsingular if and only if

$$
\begin{gathered}
r\left(H_{1}\right)-2 r\left(A_{1}\right)-2 r\left(A_{2}\right) \geq p, \\
r\left(H_{2}\right)-2 r\left(B_{1}\right)-2 r\left(A_{2}\right) \geq p, \\
r\left(H_{3}\right)-2 r\left(A_{2}\right)-r\left(A_{1}\right)-r\left(B_{1}\right) \geq p .
\end{gathered}
$$

\section{The Solvability Conditions and the General Solution to System (6)}

We now turn our attention to (6). We in this section use Theorem 9 to give some necessary and sufficient conditions for the existence of a solution to (6) and present an expression of the general solution to (6). We begin with a lemma which is used in the latter part of this section.

Lemma 11 (see [14]). Let $A_{1} \in \mathbb{C}^{m \times n_{1}}, B_{1} \in \mathbb{C}^{m \times n_{2}}, C_{1} \in$ $\mathbb{C}^{q \times m}$, and $E_{1} \in \mathbb{C}_{h}^{m \times m}$ be given. Let $A=R_{A_{1}} B_{1}, B=C_{1} R_{A_{1}}$, $E=R_{A_{1}} E_{1} R_{A_{1}}, M=R_{A} B^{*}, N=A^{*} L_{B}$, and $S=B^{*} L_{M}$. Then the following statements are equivalent:

(1) equation (5) is consistent,

(2)

$$
R_{M} R_{A} E=0, \quad R_{A} E R_{A}=0, \quad L_{B} E L_{B}=0,
$$

(3)

$$
\begin{gathered}
r\left[\begin{array}{cccc}
E_{1} & B_{1} & C_{1}^{*} & A_{1} \\
A_{1}^{*} & 0 & 0 & 0
\end{array}\right]=r\left[\begin{array}{lll}
B_{1} & C_{1}^{*} & A_{1}
\end{array}\right]+r\left(A_{1}\right), \\
r\left[\begin{array}{ccc}
E_{1} & B_{1} & A_{1} \\
A_{1}^{*} & 0 & 0 \\
B_{1}^{*} & 0 & 0
\end{array}\right]=2 r\left[\begin{array}{ll}
B_{1} & A_{1}
\end{array}\right] \\
r\left[\begin{array}{ccc}
E_{1} & C_{1}^{*} & A_{1} \\
A_{1}^{*} & 0 & 0 \\
C_{1} & 0 & 0
\end{array}\right]=2 r\left[\begin{array}{ll}
C_{1}^{*} & A_{1}
\end{array}\right] .
\end{gathered}
$$

In this case, the general solution of (5) can be expressed as

$$
\begin{aligned}
Y= & \frac{1}{2}\left[A^{\dagger} E B^{\dagger}-A^{\dagger} B^{*} M^{\dagger} E B^{\dagger}-A^{\dagger} S\left(B^{\dagger}\right)^{*} E N^{\dagger} A^{*} B^{\dagger}\right. \\
& \left.+A^{\dagger} E\left(M^{\dagger}\right)^{*}+\left(N^{\dagger}\right)^{*} E B^{\dagger} S^{\dagger} S\right]+L_{A} V_{1}+V_{2} R_{B} \\
& +U_{1} L_{S} L_{M}+R_{N} U_{2}^{*} L_{M}-A^{\dagger} S U_{2} R_{N} A^{*} B^{\dagger}, \\
X= & A_{1}^{\dagger}\left[E_{1}-B_{1} Y C_{1}-\left(B_{1} Y C_{1}\right)^{*}\right] \\
& -\frac{1}{2} A_{1}^{\dagger}\left[E_{1}-B_{1} Y C_{1}-\left(B_{1} Y C_{1}\right)^{*}\right] A_{1} A_{1}^{\dagger} \\
& -A_{1}^{\dagger} W_{1} A_{1}^{*}+W_{1}^{*} A_{1} A_{1}^{\dagger}+L_{A_{1}} W_{2},
\end{aligned}
$$

where $U_{1}, U_{2}, V_{1}, V_{2}, W_{1}$, and $W_{2}$ are arbitrary matrices over $\mathbb{C}$ with appropriate sizes.

Now we give the main theorem of this section.

Theorem 12. Let $A_{i}, C_{i},(i=1,2,3), B_{j}$, and $D_{j},(j=1,3)$ be given. Set

$$
\begin{gathered}
A=B_{3} L_{A_{2}}, \quad B=C_{3} L_{A_{1}}, \\
C=R_{B_{1}} D_{3}, \quad F=R_{A} B, \\
G=C R_{A}, \quad M=R_{F} G^{*}, \\
N=F^{*} L_{G}, \quad S=G^{*} L_{M}, \\
D=A_{3}-B_{3} A_{2}^{\dagger} C_{2}-\left(B_{3} A_{2}^{\dagger} C_{2}\right)^{*} \\
-C_{3}\left(A_{1}^{\dagger} C_{1}+L_{A_{1}} D_{1} B_{1}^{\dagger}\right) D_{3} \\
-D_{3}^{*}\left(A_{1}^{\dagger} C_{1}+L_{A_{1}} D_{1} B_{1}^{\dagger}\right)^{*} C_{3}^{*}, \\
E=R_{A} D R_{A} .
\end{gathered}
$$

Then the following statements are equivalent:

(1) system (6) is consistent,

(2) the equalities in (14) and (17) hold, and

$$
R_{M} R_{F} E=0, \quad R_{F} E R_{F}=0, \quad L_{G} E L_{G}=0,
$$

(3) the equalities in (15) and (18) hold, and

$$
\begin{gathered}
{\left[\begin{array}{ccccc}
A_{3} & C_{3} & D_{3}^{*} & B_{3} & C_{2}^{*} \\
B_{3}^{*} & 0 & 0 & 0 & A_{2}^{*} \\
C_{1} D_{3} & A_{1} & 0 & 0 & 0 \\
D_{1}^{*} C_{3}^{*} & 0 & B_{1}^{*} & 0 & 0 \\
C_{2} & 0 & 0 & A_{2} & 0
\end{array}\right]=r\left[\begin{array}{ccc}
C_{3} & D_{3}^{*} & B_{3} \\
A_{1} & 0 & 0 \\
0 & B_{1}^{*} & 0 \\
0 & 0 & A_{2}
\end{array}\right]+r\left[\begin{array}{l}
A_{2} \\
B_{3}
\end{array}\right],} \\
r\left[\begin{array}{ccccc}
A_{3} & C_{3} & B_{3} & D_{3}^{*} C_{1}^{*} & C_{2}^{*} \\
C_{3}^{*} & 0 & 0 & A_{1}^{*} & 0 \\
B_{3}^{*} & 0 & 0 & 0 & A_{2}^{*} \\
C_{1} D_{3} & A_{1} & 0 & 0 & 0 \\
C_{2} & 0 & A_{2} & 0 & 0
\end{array}\right]=2 r\left[\begin{array}{cc}
C_{3} & B_{3} \\
A_{1} & 0 \\
0 & A_{2}
\end{array}\right] \\
r\left[\begin{array}{ccccc}
A_{3} & D_{3}^{*} & B_{3} & C_{3} D_{1} & C_{2}^{*} \\
D_{3} & 0 & 0 & B_{1} & 0 \\
B_{3}^{*} & 0 & 0 & 0 & A_{2}^{*} \\
D_{1}^{*} C_{3}^{*} & B_{1}^{*} & 0 & 0 & 0 \\
C_{2} & 0 & A_{2} & 0 & 0
\end{array}\right]=2 r\left[\begin{array}{cc}
D_{3}^{*} & B_{3} \\
B_{1}^{*} & 0 \\
0 & A_{2}
\end{array}\right]
\end{gathered}
$$

In this case, the general solution of system (6) can be expressed as

$$
\begin{gathered}
X=A_{2}^{\dagger} C_{2}+L_{A_{2}} U \\
Y=A_{1}^{\dagger} C_{1}+L_{A_{1}} D_{1} B_{1}^{\dagger}+L_{A_{1}} V R_{B_{1}}
\end{gathered}
$$


where

$$
\begin{aligned}
V=\frac{1}{2}[ & F^{\dagger} E G^{\dagger}-F^{\dagger} G^{*} M^{\dagger} E G^{\dagger}-F^{\dagger} S\left(G^{\dagger}\right)^{*} E N^{\dagger} F^{*} G^{\dagger} \\
& \left.+F^{\dagger} E\left(M^{\dagger}\right)^{*}+\left(N^{\dagger}\right)^{*} E G^{\dagger} S^{\dagger} S\right]+L_{F} V_{1} \\
& +V_{2} R_{G}+U_{1} L_{S} L_{M}+R_{N} U_{2}^{*} L_{M}-F^{\dagger} S U_{2} R_{N} F^{*} G^{\dagger}, \\
U= & A^{\dagger}\left[D-B V C-(B V C)^{*}\right] \\
& -\frac{1}{2} A^{\dagger}\left[D-B V C-(B V C)^{*}\right] A A^{\dagger} \\
- & A^{\dagger} W_{1} A^{*}+W_{1}^{*} A A^{\dagger}+L_{A} W_{2},
\end{aligned}
$$

where $U_{1}, U_{2}, V_{1}, V_{2}, W_{1}$, and $W_{2}$ are arbitrary matrices over $\mathbb{C}$ with appropriate sizes.

Proof. (2) $\Leftrightarrow$ (3): Applying Lemma 3 and Lemma 11 gives

$$
\begin{aligned}
& R_{M} R_{F} E=0 \Longleftrightarrow r\left(R_{M} R_{F} E\right) \\
& =0 \Longleftrightarrow r\left[\begin{array}{cccc}
D & B & C^{*} & A \\
A^{*} & 0 & 0 & 0
\end{array}\right] \\
& =r\left[\begin{array}{lll}
B & C^{*} & A
\end{array}\right]+r(A) \\
& \Longleftrightarrow r\left[\begin{array}{ccccc}
D & C_{3} & D_{3}^{*} & B_{3} & 0 \\
B_{3}^{*} & 0 & 0 & 0 & A_{2}^{*} \\
0 & A_{1} & 0 & 0 & 0 \\
0 & 0 & B_{1}^{*} & 0 & 0 \\
0 & 0 & 0 & A_{2} & 0
\end{array}\right] \\
& =r\left[\begin{array}{ccc}
C_{3} & D_{3}^{*} & B_{3} \\
A_{1} & 0 & 0 \\
0 & B_{1}^{*} & 0 \\
0 & 0 & A_{2}
\end{array}\right]+r\left[\begin{array}{l}
A_{2} \\
B_{3}
\end{array}\right] \\
& \Longleftrightarrow r\left[\begin{array}{ccccc}
A_{3} & C_{3} & D_{3}^{*} & B_{3} & C_{2}^{*} \\
B_{3}^{*} & 0 & 0 & 0 & A_{2}^{*} \\
C_{1} D_{3} & A_{1} & 0 & 0 & 0 \\
D_{1}^{*} C_{3}^{*} & 0 & B_{1}^{*} & 0 & 0 \\
C_{2} & 0 & 0 & A_{2} & 0
\end{array}\right] \\
& =r\left[\begin{array}{ccc}
C_{3} & D_{3}^{*} & B_{3} \\
A_{1} & 0 & 0 \\
0 & B_{1}^{*} & 0 \\
0 & 0 & A_{2}
\end{array}\right]+r\left[\begin{array}{l}
A_{2} \\
B_{3}
\end{array}\right] \text {. }
\end{aligned}
$$

By a similar approach, we can obtain that

$$
\begin{aligned}
R_{F} E R_{F} & =0 \Longleftrightarrow r\left[\begin{array}{ccccc}
A_{3} & C_{3} & B_{3} & D_{3}^{*} C_{1}^{*} & C_{2}^{*} \\
C_{3}^{*} & 0 & 0 & A_{1}^{*} & 0 \\
B_{3}^{*} & 0 & 0 & 0 & A_{2}^{*} \\
C_{1} D_{3} & A_{1} & 0 & 0 & 0 \\
C_{2} & 0 & A_{2} & 0 & 0
\end{array}\right] \\
& =2 r\left[\begin{array}{cc}
C_{3} & B_{3} \\
A_{1} & 0 \\
0 & A_{2}
\end{array}\right],
\end{aligned}
$$

$$
\begin{aligned}
L_{G} E L_{G} & =0 \Longleftrightarrow r\left[\begin{array}{ccccc}
A_{3} & D_{3}^{*} & B_{3} & C_{3} D_{1} & C_{2}^{*} \\
D_{3} & 0 & 0 & B_{1} & 0 \\
B_{3}^{*} & 0 & 0 & 0 & A_{2}^{*} \\
D_{1}^{*} C_{3}^{*} & B_{1}^{*} & 0 & 0 & 0 \\
C_{2} & 0 & A_{2} & 0 & 0
\end{array}\right] \\
& =2 r\left[\begin{array}{cc}
D_{3}^{*} & B_{3} \\
B_{1}^{*} & 0 \\
0 & A_{2}
\end{array}\right] .
\end{aligned}
$$

(1) $\Leftrightarrow(2)$ : We separate the four equations in system (6) into three groups:

$$
\begin{gathered}
A_{1} Y=C_{1}, \quad Y B_{1}=D_{1}, \\
A_{2} X=C_{2}, \\
B_{3} X+\left(B_{3} X\right)^{*}+C_{3} Y D_{3}+\left(C_{3} Y D_{3}\right)^{*}=A_{3} .
\end{gathered}
$$

By Lemma 1, we obtain that system (80) is solvable if and only if (14), (81) is consistent if and only if (17). The general solutions to system (80) and (81) can be expressed as (16) and (19), respectively. Substituting (16) and (19) into (82) yields

$$
A U+(A U)^{*}+B V C+(B V C)^{*}=D .
$$

Hence, the system (5) is consistent if and only if (80), (81), and (83) are consistent, respectively. It follows from Lemma 11 that (83) is solvable if and only if

$$
R_{M} R_{F} E=0, \quad R_{F} E R_{F}=0, \quad L_{G} E L_{G}=0 .
$$

We know by Lemma 11 that the general solution of (83) can be expressed as (77).

In Theorem 12, let $A_{1}$ and $D_{1}$ vanish. Then we can obtain the general solution to the following system:

$$
\begin{gathered}
A_{2} X=C_{2}, \quad Y B_{1}=D_{1}, \\
B_{3} X+\left(B_{3} X\right)^{*}+C_{3} Y D_{3}+\left(C_{3} Y D_{3}\right)^{*}=A_{3} .
\end{gathered}
$$

Corollary 13. Let $A_{2}, C_{2}, B_{1}, D_{1}, B_{3}, C_{3}, D_{3}$, and $A_{3}=A_{3}^{*}$ be given. Set

$$
\begin{gathered}
A=B_{3} L_{A_{2}}, \quad C=R_{B_{1}} D_{3}, \\
F=R_{A} C_{3}, \quad G=C R_{A}, \\
M=R_{F} G^{*}, \quad N=F^{*} L_{G}, \\
S=G^{*} L_{M}, \\
D=A_{3}-B_{3} A_{2}^{\dagger} C_{2}-\left(B_{3} A_{2}^{\dagger} C_{2}\right)^{*} \\
-C_{3} D_{1} B_{1}^{\dagger} D_{3}-\left(C_{3} D_{1} B_{1}^{\dagger} D_{3}\right)^{*}, \\
E=R_{A} D R_{A} .
\end{gathered}
$$


Then the following statements are equivalent:

(1) system (85) is consistent

(2)

$$
\begin{gathered}
R_{A_{2}} C_{2}=0, \quad D_{1} L_{B_{1}}=0, \quad R_{M} R_{F} E=0, \\
R_{F} E R_{F}=0, \quad L_{G} E L_{G}=0,
\end{gathered}
$$

(3)

$$
\begin{gathered}
r\left[A_{2} C_{2}\right]=r\left(A_{2}\right), \quad\left[\begin{array}{l}
D_{1} \\
B_{1}
\end{array}\right]=r\left(B_{1}\right), \\
r\left[\begin{array}{ccccc}
A_{3} & C_{3} & D_{3}^{*} & B_{3} & C_{2}^{*} \\
B_{3}^{*} & 0 & 0 & 0 & A_{2}^{*} \\
D_{1}^{*} C_{3}^{*} & 0 & B_{1}^{*} & 0 & 0 \\
C_{2} & 0 & 0 & A_{2} & 0
\end{array}\right]=r\left[\begin{array}{ccc}
C_{3} & D_{3}^{*} & B_{3} \\
0 & B_{1}^{*} & 0 \\
0 & 0 & A_{2}
\end{array}\right]+r\left[\begin{array}{l}
A_{2} \\
B_{3}
\end{array}\right], \\
r\left[\begin{array}{ccccc}
A_{3} & C_{3} & B_{3} & C_{2}^{*} \\
C_{3}^{*} & 0 & 0 & 0 \\
B_{3}^{*} & 0 & 0 & A_{2}^{*} \\
C_{2} & 0 & A_{2} & 0
\end{array}\right]=2 r\left[\begin{array}{cc}
C_{3} & B_{3} \\
0 & A_{2}
\end{array}\right], \\
{\left[\begin{array}{ccccc}
A_{3} & D_{3}^{*} & B_{3} & C_{3} D_{1} & C_{2}^{*} \\
D_{3} & 0 & 0 & B_{1} & 0 \\
B_{3}^{*} & 0 & 0 & 0 & A_{2}^{*} \\
D_{1}^{*} C_{3}^{*} & B_{1}^{*} & 0 & 0 & 0 \\
C_{2} & 0 & A_{2} & 0 & 0
\end{array}\right]=2 r\left[\begin{array}{cc}
D_{3}^{*} & B_{3} \\
B_{1}^{*} & 0 \\
0 & A_{2}
\end{array}\right]}
\end{gathered}
$$

In this case, the general solution of system (6) can be expressed as

$$
\begin{aligned}
& X=A_{2}^{\dagger} C_{2}+L_{A_{2}} U, \\
& Y=D_{1} B_{1}^{\dagger}+V R_{B_{1}},
\end{aligned}
$$

where

$$
\begin{aligned}
V=\frac{1}{2}[ & F^{\dagger} E G^{\dagger}-F^{\dagger} G^{*} M^{\dagger} E G^{\dagger}-F^{\dagger} S\left(G^{\dagger}\right)^{*} E N^{\dagger} F^{*} G^{\dagger} \\
& \left.+F^{\dagger} E\left(M^{\dagger}\right)^{*}+\left(N^{\dagger}\right)^{*} E G^{\dagger} S^{\dagger} S\right]+L_{F} V_{1} \\
& +V_{2} R_{G}+U_{1} L_{S} L_{M}+R_{N} U_{2}^{*} L_{M}-F^{\dagger} S U_{2} R_{N} F^{*} G^{\dagger}, \\
U= & A^{\dagger}\left[D-C_{3} V C-\left(C_{3} V C\right)^{*}\right] \\
& -\frac{1}{2} A^{\dagger}\left[D-C_{3} V C-\left(C_{3} V C\right)^{*}\right] A A^{\dagger} \\
& -A^{\dagger} W_{1} A^{*}+W_{1}^{*} A A^{\dagger}+L_{A} W_{2},
\end{aligned}
$$

where $U_{1}, U_{2}, V_{1}, V_{2}, W_{1}$, and $W_{2}$ are arbitrary matrices over $\mathbb{C}$ with appropriate sizes.

\section{Acknowledgments}

The authors would like to thank Dr. Mohamed, Dr. Sivakumar, and a referee very much for their valuable suggestions and comments, which resulted in a great improvement of the original paper. This research was supported by the Grants from the National Natural Science Foundation of China (NSFC (11161008)) and Doctoral Program Fund of Ministry of Education of P.R.China (20115201110002).

\section{References}

[1] H. Zhang, "Rank equalities for Moore-Penrose inverse and Drazin inverse over quaternion," Annals of Functional Analysis, vol. 3, no. 2, pp. 115-127, 2012.

[2] L. Bao, Y. Lin, and Y. Wei, "A new projection method for solving large Sylvester equations," Applied Numerical Mathematics, vol. 57, no. 5-7, pp. 521-532, 2007.

[3] M. Dehghan and M. Hajarian, "On the generalized reflexive and anti-reflexive solutions to a system of matrix equations," Linear Algebra and Its Applications, vol. 437, no. 11, pp. 2793-2812, 2012.

[4] F. O. Farid, M. S. Moslehian, Q.-W. Wang, and Z.-C. Wu, "On the Hermitian solutions to a system of adjointable operator equations," Linear Algebra and Its Applications, vol. 437, no. 7, pp. 1854-1891, 2012.

[5] Z. H. He and Q. W. Wang, "A real quaternion matrix equation with with applications," Linear Multilinear Algebra, vol. 61, pp. 725-740, 2013.

[6] Q.-W. Wang and Z.-C. Wu, "Common Hermitian solutions to some operator equations on Hilbert $C^{x}$-modules," Linear Algebra and Its Applications, vol. 432, no. 12, pp. 3159-3171, 2010.

[7] Q.-W. Wang and C.-Z. Dong, "Positive solutions to a system of adjointable operator equations over Hilbert $C^{x}$-modules," Linear Algebra and Its Applications, vol. 433, no. 7, pp. 1481-1489, 2010.

[8] Q.-W. Wang, J. W. van der Woude, and H.-X. Chang, "A system of real quaternion matrix equations with applications," Linear Algebra and Its Applications, vol. 431, no. 12, pp. 2291-2303, 2009.

[9] Q. W. Wang, H.-S. Zhang, and G.-J. Song, "A new solvable condition for a pair of generalized Sylvester equations," Electronic Journal of Linear Algebra, vol. 18, pp. 289-301, 2009.

[10] H. W. Braden, “The equations $A^{T} X \pm X^{T} A=B$," SIAM Journal on Matrix Analysis and Applications, vol. 20, no. 2, pp. 295-302, 1999.

[11] D. S. Djordjević, "Explicit solution of the operator equation $A^{*} X+X^{ \pm} A=B$," Journal of Computational and Applied Mathematics, vol. 200, no. 2, pp. 701-704, 2007.

[12] W. Cao, "Solvability of a quaternion matrix equation," Applied Mathematics, vol. 17, no. 4, pp. 490-498, 2002.

[13] Q. Xu, L. Sheng, and Y. Gu, "The solutions to some operator equations," Linear Algebra and Its Applications, vol. 429, no. 8-9, pp. 1997-2024, 2008.

[14] Q.-W. Wang and Z.-H. He, "Some matrix equations with applications," Linear and Multilinear Algebra, vol. 60, no. 11-12, pp. 1327-1353, 2012.

[15] Y. Tian, "Maximization and minimization of the rank and inertia of the Hermitian matrix expression $A-B X-(B X)^{x}$," Linear Algebra and Its Applications, vol. 434, no. 10, pp. 21092139, 2011.

[16] Z.-H. He and Q.-W. Wang, "Solutions to optimization problems on ranks and inertias of a matrix function with applications," Applied Mathematics and Computation, vol. 219, no. 6, pp. 29893001, 2012.

[17] Y. Liu and Y. Tian, "Max-min problems on the ranks and inertias of the matrix expressions $A-B X C \pm(B X C)^{*}$," Journal of 
Optimization Theory and Applications, vol. 148, no. 3, pp. 593622, 2011.

[18] D. Chu, Y. S. Hung, and H. J. Woerdeman, "Inertia and rank characterizations of some matrix expressions," SIAM Journal on Matrix Analysis and Applications, vol. 31, no. 3, pp. 1187-1226, 2009.

[19] Y. Liu and Y. Tian, "A simultaneous decomposition of a matrix triplet with applications," Numerical Linear Algebra with Applications, vol. 18, no. 1, pp. 69-85, 2011.

[20] X. Zhang, Q.-W. Wang, and X. Liu, "Inertias and ranks of some Hermitian matrix functions with applications," Central European Journal of Mathematics, vol. 10, no. 1, pp. 329-351, 2012.

[21] Q.-W. Wang, "The general solution to a system of real quaternion matrix equations," Computers \& Mathematics with Applications, vol. 49, no. 5-6, pp. 665-675, 2005.

[22] Y. Tian, "Equalities and inequalities for inertias of Hermitian matrices with applications," Linear Algebra and Its Applications, vol. 433, no. 1, pp. 263-296, 2010.

[23] G. Marsaglia and G. P. H. Styan, "Equalities and inequalities for ranks of matrices," Linear and Multilinear Algebra, vol. 2, pp. 269-292, 1974. 


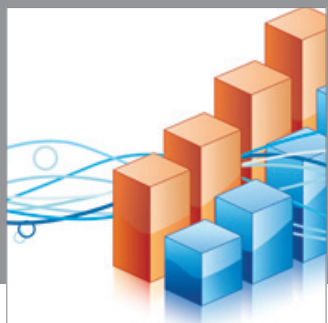

Advances in

Operations Research

mansans

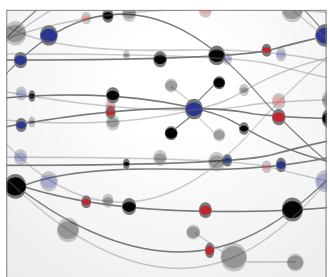

The Scientific World Journal
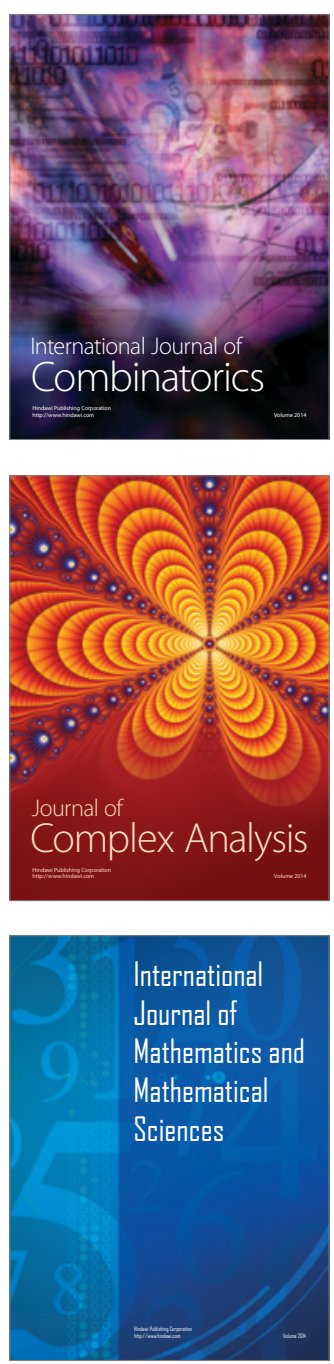
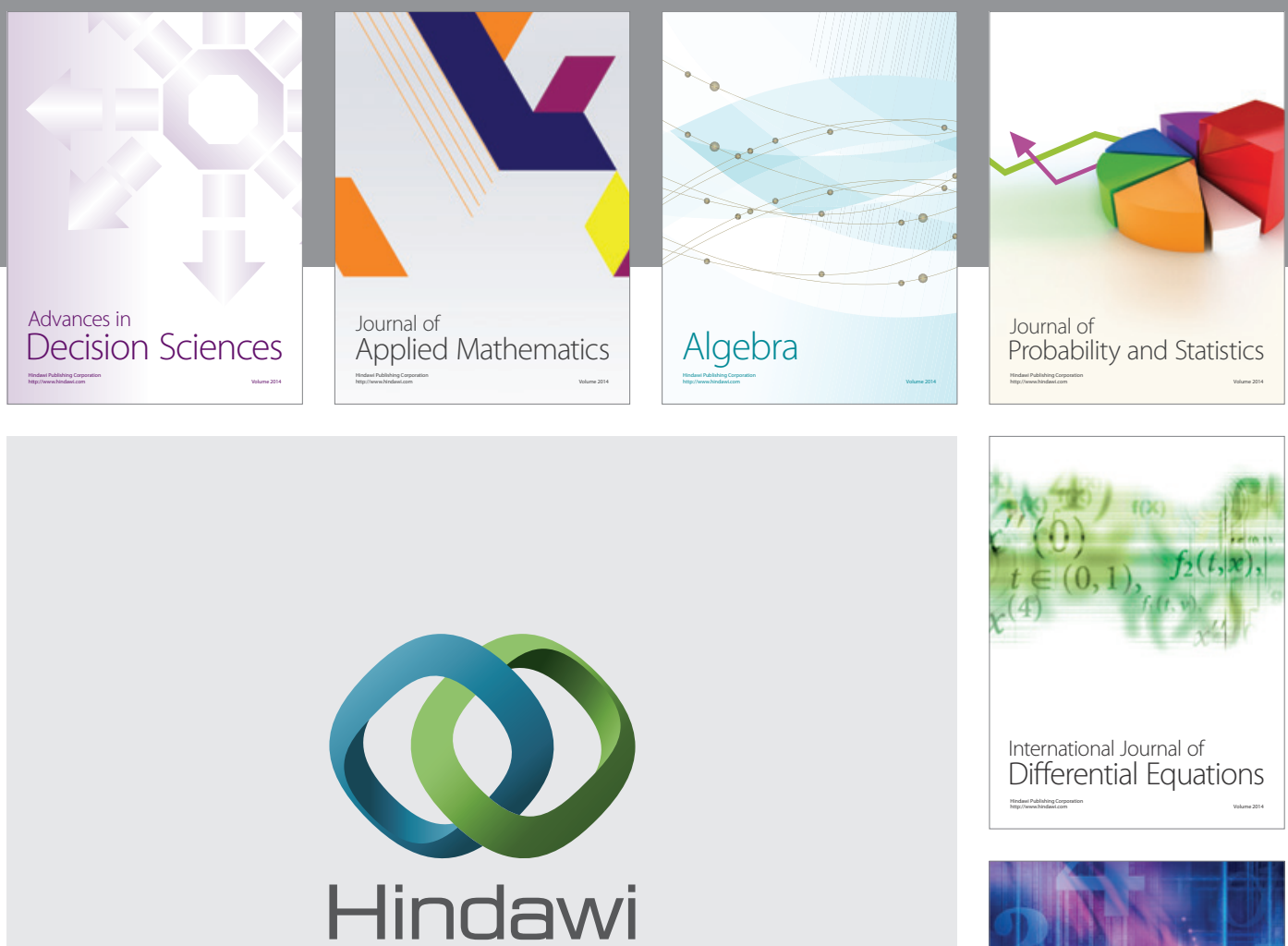

Submit your manuscripts at http://www.hindawi.com
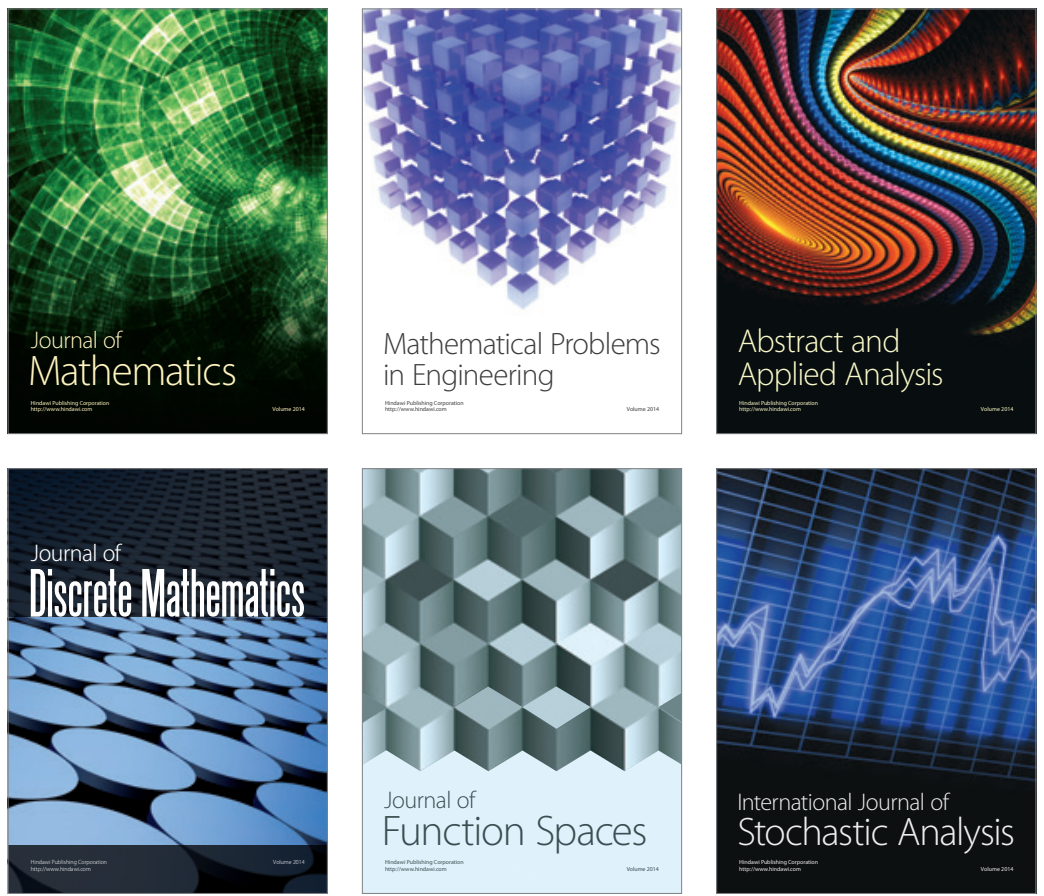

Journal of

Function Spaces

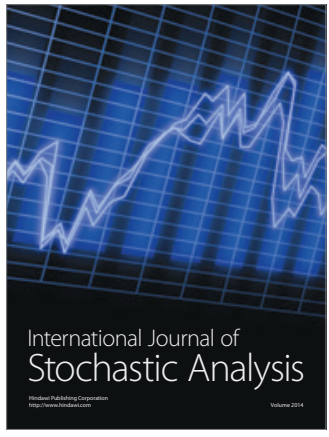

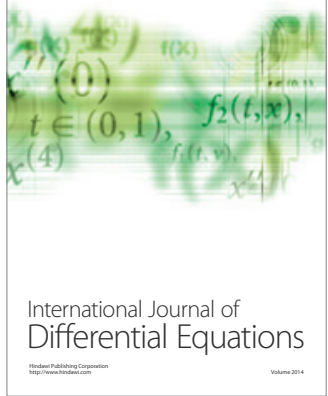
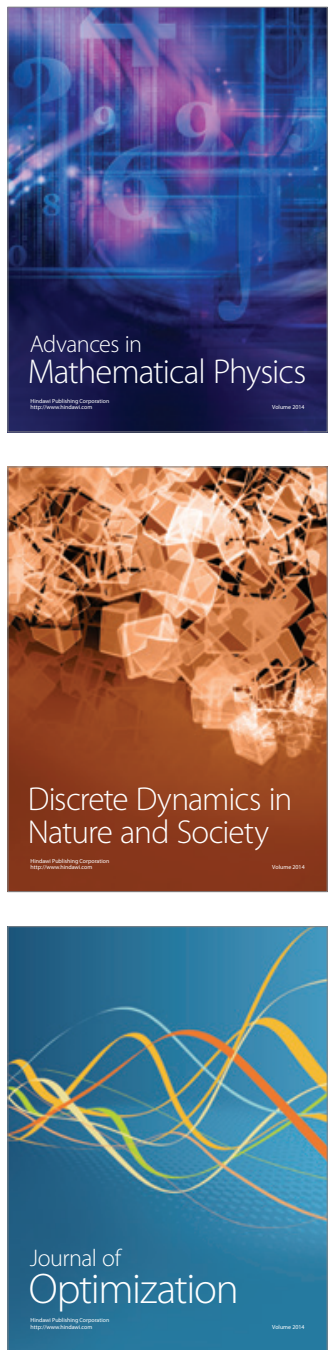